\title{
Optimizing Self-wicking Grids for Chameleon
}

Hui Wei ${ }^{1}$, Dea Kurti ${ }^{1}$, William C. Budell ${ }^{1}$, Venkata P. Dandey ${ }^{1}$, Clinton S. Potter ${ }^{1,2}$, Bridget Carragher ${ }^{1,2}$

${ }^{1}$ National Resource for Automated Molecular Microscopy, Simons Electron Microscopy Center, New York Structural Biology Center, New York, United States, ${ }^{2}$ Department of Biochemistry and Molecular Biophysics, Columbia University, New York, New York, United States

Chameleon (SPT Labtech Ltd.) is a commercial instrument based on the Spotiton technology [1][2] that uses piezo dispensing of picoliter volumes onto "self-blotting" nanowire grids [3] to vitrify sample for cryo electron microscopy (cryoEM). This differs from traditional methods for vitrification where microliters of samples are applied to a grid and reduced to a thin film using externally applied blotting paper. The chameleon approach has three main advantages. (i) Sample is applied as the grid moves rapidly past the piezo tip en route to vitrification in ethane. This reduces the time that particles are in a thin film and thus exposed to the deleterious effects of interactions with the air-water interface. (ii) Sample volume used to make each grid is $<0.01 \mu \mathrm{L}$, and thus large numbers of grids can be made from a starting volume of $3 \mu \mathrm{L}$, compared to only a single grid using traditional methods. (iii) Several cameras built into the system provide visual feedback to evaluate the potential quality of a grid before transferring it to the electron microscope.

Decreasing the time between spot and plunge increases the benefits of limiting exposure to the air-water interface [4] but creates problems with achieving an even and thin layer of vitreous ice across each grid square. For example, at spot to plunge times of $133 \mathrm{~ms}$ we frequently observe that ice across holes in the center of a grid square is thicker than in the holes at the edge; these edge holes can also be completely dry (see Fig. 1).

There are two main factors that influence ice distribution and thickness. First, the supporting substrate material - most commonly used are carbon and gold. Carbon substrates generally result in thicker ice compared to gold substrates when other conditions are equal. Second, grid preparation - using either plasma cleaning or glow discharge - has a major effect on the ice thickness. Too little plasma cleaning results in very slow wicking and overall too thick ice, whereas a high dose of plasma often results in the phenomenon of thick ice at the center and empty holes at the edge of the square. We have been experimenting with grid preparation and substrates to find conditions that provide more optimal ice (Fig. 2 and 3). These methods will be discussed and results will be presented [5].
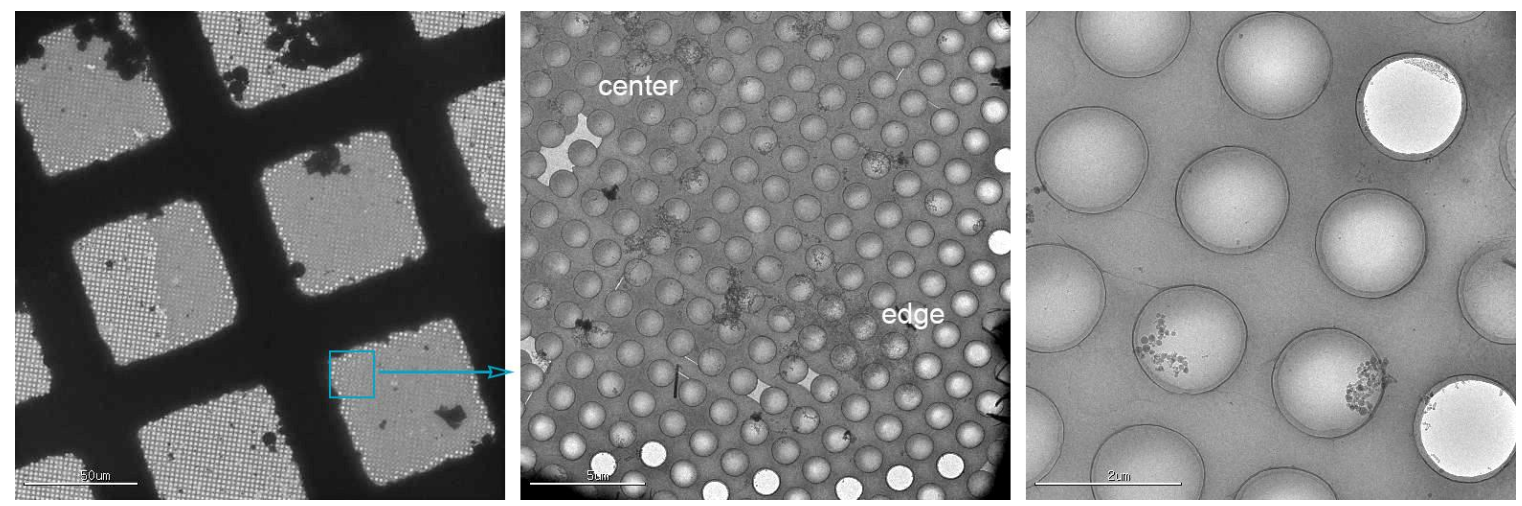

Figure 1. Very fast plunge speeds requires fast wicking but this sometimes leads to uneven ice distribution and thickness across each grid square. In this case holes near the edge of the square are empty or much thinner than in the middle of the square. 

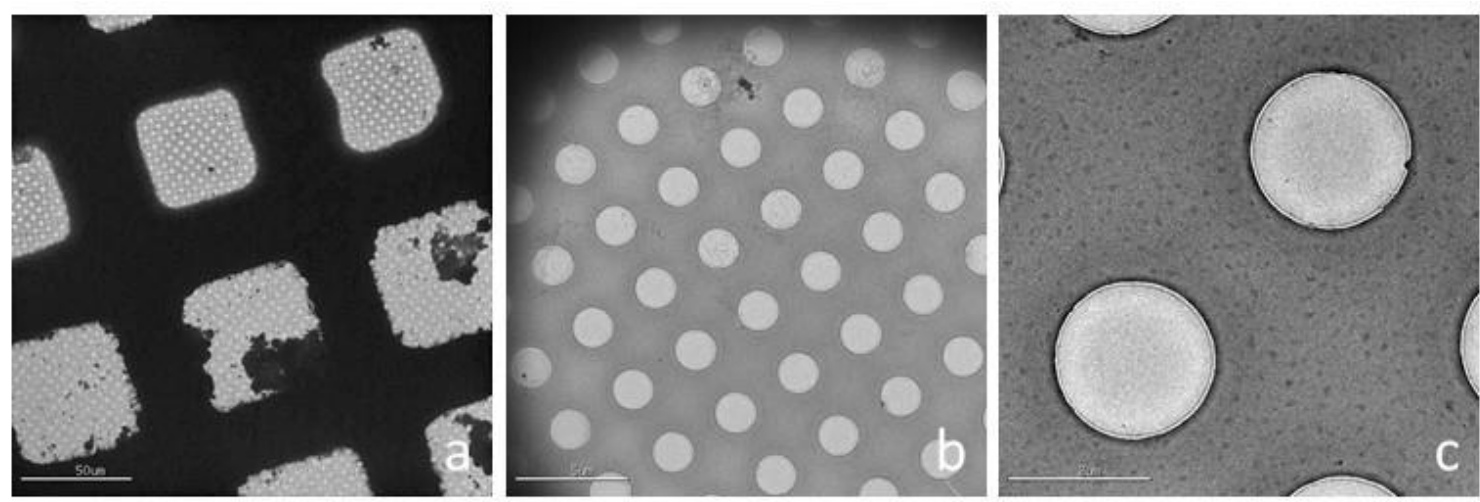

Fig 2: Continues wicking made the ice layer thin and even. a) No dry area at the edge of the square. b) Even ice at the center and the edge of the square. c) Thin ice in the hole.

Figure 2. Modifying grid preparation conditions can result in a much more even distribution of vitreous ice across the grid square.

\section{References}

[1] Dandey et al. Spotiton: New features and applications. J Struct Biol. 2018.

[2] Razinkov et al. A new method for vitrifying samples for cryoEM. J Struct Biol. 2016;195(2):190-8.

[3] Wei et al. Optimizing "self-wicking" nanowire grids. J Struct Biol. 2018.

[4] Noble et al. Reducing effects of particle adsorption to the air-water interface in cryo-EM. Nat Methods. 2018;15(10):793-5..

[5] Work supported by grants from the Simons Foundation (SF349247), NYSTAR, and the NIH (GM103310, RR029300). 